\title{
Poor performance on cognitive tasks in depression: Doing too much or not enough?
}

\author{
Neil P. Jones And Greg J. Siegle \\ Western Psychiatric Institute and Clinic, Pittsburgh, Pennsylvania \\ and University of Pittsburgh Medical Center, Pittsburgh, Pennsylvania \\ EMILIE R. Muelly \\ Pennsylvania State University, University Park, Pennsylvania \\ AND \\ Agnes Haggerty and Frank Ghinassi \\ Western Psychiatric Institute and Clinic, Pittsburgh, Pennsylvania \\ and University of Pittsburgh Medical Center, Pittsburgh, Pennsylvania
}

\begin{abstract}
Depressed people perform poorly on cognitive tasks. It is unclear whether these deficits are due to decreased devotion of task-related resources or to increased attention to non-task-related information. In the present study, we examined the degree to which depressed and healthy adults displayed pupillary motility that varied at the frequency of presented stimuli on a cognitive task, which we interpreted as task-related processing, and at other frequencies, which we interpreted as reflecting intrinsic processing. Depressed participants made more consecutive errors than did controls. More pupillary motility at other frequencies was associated with poorer performance, whereas more pupillary motility at the frequency of presented stimuli was associated with better performance. Depressed participants had more pupillary motility at other frequencies, which partially mediated observed deficits in cognitive performance. These findings support the hypothesis that allocating cognitive resources to intrinsic processing contributes to observed cognitive deficits in depression.
\end{abstract}

Clinically depressed adults demonstrate deficits in cognitive control (Austin, Mitchell, \& Goodwin, 2001; Ottowitz, Tondo, Dougherty, \& Savage, 2002; Rogers et al., 2004). Cognitive control facilitates, among other faculties, the following: attention allocation, encoding goalrelevant information, the inhibition of processing of irrelevant information, monitoring performance, and using feedback to adjust future responding (MacDonald, Cohen, Stenger, \& Carter, 2000; Ridderinkhof, Ullsperger, Crone, $\&$ Nieuwenhuis, 2004). In particular, relative to healthy controls, depressed individuals demonstrate abnormal (either hyper- or hypo-) activity in neural regions underlying cognitive control and poor behavioral performance during demanding cognitive tasks (Holmes \& Pizzagalli, 2008; Okada, Okamoto, Morinobu, \& Yamawaki, 2003; Pizzagalli, Peccoralo, Davidson, \& Cohen, 2006).

The reasons for poor performance during cognitive tasks in depression are unclear. The tendency to engage in intrinsic processing - for example, focusing on negative automatic thoughts - has been hypothesized to use cognitive resources that would otherwise be allocated to a cognitive task resulting in poor performance (Christopher \& MacDonald, 2005; Holmes \& Pizzagalli, 2007, 2008). Consistent with this hypothesis, relative to controls, depressed participants demonstrate a hyperactivation in neural regions that are implicated in affective processing, such as the rostral anterior cingulate, after making an initial error on a demanding cognitive task, which is subsequently associated with a failure to recruit dorsolateral prefrontal cortex (DLPFC) based cognitive control and poorer subsequent performance on the task (Holmes \& Pizzagalli, 2008). Similarly, depressed participants who were experimentally induced to ruminate - that is, to repetitively focus on themselves and the nature and implications of their negative feelings (Nolen-Hoeksema, 1991; Watkins \& Brown, 2002), prior to engaging in a cognitive task - experienced more sadness and intrinsic intrusive thoughts and produced more errors than they did when they were induced to engage in distraction by focusing on external information unrelated to symptoms or feelings (Watkins \& Brown, 2002). Furthermore, depressed participants demonstrate difficulty inhibiting irrelevant negative material from working memory (Joormann \& Gotlib, 2008). Thus, studies could suggest that depressed individuals engage in increased intrinsic processing that potentially depletes or taxes the ability to engage in controlled processing of information.

Alternately, evidence suggests that poor cognitive performance may be more related to a lack of cognitive re- 
sources that are necessary to do the task independent of engaging in intrinsic processing. Some investigations have demonstrated deficits in behavioral performance, which coincides with decreased neural activation in brain regions that are critical for cognitive control in the absence of activity in neural regions that are implicated in emotional processing (Audenaert et al., 2002; Elliott, Baker, et al., 1997; Okada et al., 2003). Depressed adults, relative to controls, have also demonstrated abnormal (either hyperor hypo-) activity in dorsolateral prefrontal regions on cognitive tasks in the presence of intact behavioral performance (Fitzgerald et al., 2008; Harvey et al., 2005; Siegle, Thompson, Carter, Steinhauer, \& Thase, 2007). Although the directionality of these findings is mixed, the consistent identification of this region in relevant studies suggests a fundamental impairment that is potentially associated with an inefficiency or a lack of prefrontal resources in depression. Recent studies specifically suggested that the directionality of the abnormality may be clarified by controlling for performance, so that depressed participants display increased DLPFC in order to maintain the same degree of task performance as controls across increasing levels of task difficulty (Matsuo et al., 2007; Walter, Wolf, Spitzer, \& Vasic, 2007), although this finding must be replicated. Potentially, it is still possible for depressed persons to recruit their remaining resources to compensate for overall deficits in cognitive control. In contrast, when cognitive control resources are significantly taxed during difficult tasks, depressed individuals cannot compensate because all cognitive resources are being used (Walter et al., 2007). These findings suggest that cognitive deficits in depression could be a result of a decreased ability to allocate cognitive resources to demanding tasks.

In summary, it is unclear whether naturally occurring intrinsic information processing is associated with decreased performance above and beyond task-related processing. However, given that depression is associated with elevated negative cognitions and rumination (Riso et al., 2003; Siegle, Moore, \& Thase, 2004), it is highly unlikely that these emotional and cognitive processes cease to occur when depressed individuals engage in activities that require cognitive control in their everyday life. Thus, we favor the hypothesis that engaging in intrinsic processing is likely to deplete or tax the ability to engage in controlled processing of information. Therefore, in the present study, we examined the degree to which controlled processing deficits in depression are associated with physiological indicators of both task-related and intrinsic processing during the paced auditory serial addition task (PASAT; Gronwall, 1977).

The PASAT was chosen because it explicitly assesses information processing capacity and yields a high proportion of errors (Tombaugh, 2006). During the PASAT, individuals hear a string of digits and must sequentially compute the sum of the two previously presented digits. Thus, completion of the PASAT requires continuous controlled processing. The task recruits dorsal neural systems that have been previously implicated in cognitive control (Lazeron, Rombouts, de Sonneville, Barkhof, \& Scheltens, 2003). Trait ruminators are particularly vulnerable to experiencing increased frustration and irritation during the PASAT (Feldner, Leen-Feldner, Zvolensky, \& Lejuez, 2006); increased levels of anger and frustration induced by the PASAT are associated with lower performance in healthy, nondepressed individuals (Gow \& Deary, 2004). Thus, the PASAT seemed a particularly apt task for assessing whether task-related and intrinsic processing contribute independently to performance in depressed individuals. We hypothesized that clinically depressed individuals may be especially likely to ruminate in response to failure during the task and may thus be particularly vulnerable to poor performance, particularly involving the commission of consecutive errors due to devoting processing resources to intrinsically generated cognitions and emotions.

In the present study, we therefore used consecutive errors on the PASAT as a general marker of cognitive performance and as a potential index of the impact of interfering intrinsic cognitions and emotions (Ratcliff, 1976). We examined pupillary responses as a psychophysiological index of resource allocation (Fish \& Granholm, 2008; Granholm, Asarnow, Sarkin, \& Dykes, 1996). Pupillary motility reflects activity in a variety of brain regions that are involved in information processing (e.g., sensory, attention, memory, or motor output), including regions that subserve emotion (Koikegami \& Yoshida, 1953). Of particular importance for the present study, neuroimaging studies in which pupillary motility is measured consistently demonstrate associations of pupil dilation and activity in prefrontal regions (Johnstone, van Reekum, Urry, Kalin, \& Davidson, 2007; Siegle, Steinhauer, Stenger, Konecky, \& Carter, 2003). Pupil size increases with processing demands and in response to emotional information (for reviews, see Beatty, 1982b; Steinhauer \& Hakerem, 1992). Furthermore, pupil dilation persists if the demand is sustained (Beatty, 1982a). However, the pupil will only increase systematically with increasing processing demands that are below absolute resource limits; it reaches an asymptote when it is at or near resource limits, and it declines when processing demands exceed available resources (Granholm et al., 1996). These findings highlight that the ability to perform a task depends on the degree to which processing resources can be kept at or above a certain threshold. When two tasks are simultaneously competing for the same cognitive resources, the ability to successfully engage in both tasks depends on the degree to which resources can be kept at or above both tasks' required thresholds (Wickens, 2002). Thus, to fully understand performance on both tasks requires measuring the degree to which resources are being allocated to each task (Salvucci \& Taatgen, 2008). In addition, considering simultaneously the degree to which cognitive resources are being allocated to both tasks provides a better estimate of how close individuals are to having processing demands exceed available resources.

Since the pupil is expected to index resource allocation to both task-relevant (e.g., auditory encoding of information) and intrinsic (e.g., "I failed at this task like I fail at everything else") processing, we worked to separate physiological indicators of these two processes. Task-relevant cognitive operations per trial during the PASAT are esti- 
mated to take approximately $1 \mathrm{sec}$ and to occur in phase with stimulus presentation (Ratcliff, 1976). Therefore, our guiding assumption is that pupillary motility occurring at the frequency of task stimuli reflects task-related cognitions (e.g., 1 stimulus every $2.4 \mathrm{sec}$, which is equivalent to $0.42 \mathrm{~Hz}$ ). Pupillary motility occurring at slower frequencies may reflect longer lasting intrinsic processes that are not associated with processing each stimulus, such as rumination, in addition to other noncognitive features, such as measurement noise.

We used a statistical technique known as wavelet decomposition to quantify pupillary motility at, and slower than, the stimulus frequency during the PASAT (Torrence \& Compo, 1998). This technique breaks down responses into those that are reliably detected to occur at the same rate at which stimuli are presented (i.e., that represent task-related responses) and those that occur at other frequencies, presumably representing less-task-related processes. Wavelet analysis of pupil dilation has been used to identify differences in cognitive states such as focused versus distracted attention and alertness versus sleepiness (Marshall, 2007; Wilhelm et al., 2001).

\section{Simulations of Pupillary Motility on the PASAT}

We conducted computer simulations to evaluate the extent to which pupillary motility would be expected to capture task-related and intrinsic processing. First, we simulated allocating resources to a task requiring cognitive processing every $2 \mathrm{sec}(0.5 \mathrm{~Hz})$. This was done by convolving a pupillary impulse response from a cued reaction time (RT) task (from Siegle, Granholm, Ingram, \& Matt, 2001) with a delta function representing events spaced $2 \mathrm{sec}$ apart. Figure 1A shows the spectral power throughout the frequency domain as a function of time in this simulation, derived via Morelet wavelet decomposition. As was expected, spectral power in the $0.5-\mathrm{Hz}$ band was observed (i.e., on-task resource allocation; $M=$ $.33, S D=.00)$, and no power was observed at other frequencies, especially those below $0.37 \mathrm{~Hz}$ (i.e., no off-task resource allocation; $M=.00, S D=.00$; Figure 1A, Figure $2 \mathrm{~A}$ ). Then, we modeled random failures to allocate resources to the task - simulating the experience of task disengagement without a corresponding increase in intrinsic processes. This was done by removing random simulated pupil responses from our first simulation. Spectral power was observed at $0.5 \mathrm{~Hz}$ during intervals representing the simulated pupil response, with gaps in power at $0.5 \mathrm{~Hz}$ during time intervals during which no pupil response occurred. This translated into decreased indices of on-task resource allocation $(M=.23, S D=.02)$, but into negligible indices of off-task resource allocation $(M=.01$, $S D=.00$; Figures $1 \mathrm{~B}$ and $2 \mathrm{~B}$ ).

We next simulated slow intrinsic processing occurring in addition to task-related processing by adding occasional 10 -sec boxcars that were convolved by the pupillary impulse response to the data from the first simulation. This simulation reflects the idea that task-related and intrinsic information processing can occur independently and do not interfere with one another. As was expected, wavelet decomposition, relative to simulations of solely doing the task and experiencing random lapses in attention, revealed comparable power at the stimulus frequency $(M=$ $.32, S D=.03)$ and an increase in power that was slower than the task frequency $(M=.21, S D=.05$; Figures $1 \mathrm{C}$ and $2 \mathrm{C}$ ).

To simulate potential interference of rumination with cognition, we simulated a situation in which slow intrinsic processing would interfere with task-related processing. This was done by removing delta functions from the first simulation (as in the second simulation) whenever random slow events were inserted (as in the third simulation), and convolving the resulting waveform with the pupillary impulse response. Wavelet analysis indicated comparable stimulus-related power to the second simulation (lapses in attention) $(M=.20, S D=.02)$ and a modest increase in power that was slower than the task frequency relative to both the first simulation (solely doing the task) and second simulation (lapses in attention) $(M=.07, S D=.02$; Figures 1D and 2D).

Finally, to examine whether this type of analysis would be confounded if depressed participants did not ruminate, but rather had systematically slowed or delayed pupillary responses to stimuli, we simulated a slowed and delayed impulse response by convolving the response employed in Figures 2A-2D with a uniform smoothing kernel and multiplying it by 2 to equate response magnitudes (Figure 2E). Using this kernel, we reran the same simulations from Figure 2A. Results, shown in Figure 2F, show that although task power decreased using this kernel, off-task power did not increase. Thus, if we empirically observe decreased power at the task frequency in depressed participants, it could be due to systematically slowed delayed responses.

Thus, simulations support the utility of wavelet decomposition for dynamic detection of independent or interacting influences of processes that are associated with task-related and intrinsic processing during a continuous cognitive task.

On the basis of the reviewed research, we predicted that depressed participants would have a greater probability of making consecutive errors than would controls. Subsequently, we attempted to determine whether differences in performance between depressed participants and controls could be accounted for by a decreased allocation of cognitive resources to doing the task and/or increased allocation of cognitive resources to largely task-unrelated processing using a novel peripheral psychophysiological measure of cognitive resource allocation.

\section{METHOD}

\section{Participants}

Participants included 19 never-depressed controls and 56 patients who were diagnosed with unipolar depression. Depressed participants were diagnosed as having a current major depressive episode via a structured clinical interview (SCID-I; First, Spitzer, Gibbon, $\&$ Williams, 1996) and were recruited from the Intensive Outpatient Program at the Western Psychiatric Institute and Clinic. Participants could not meet criteria for either current or past bipolar or psychotic disorder. In addition, participants could not meet criteria for substance-use disorders during the 3 months prior to assessment. Given the fairly severe nature of this sample, 47 participants 
A

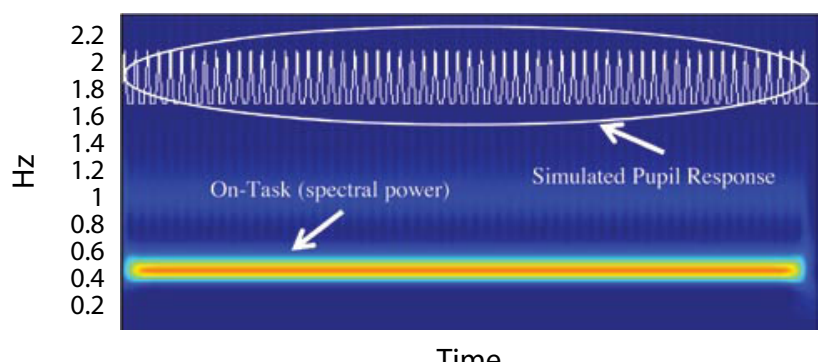

Time

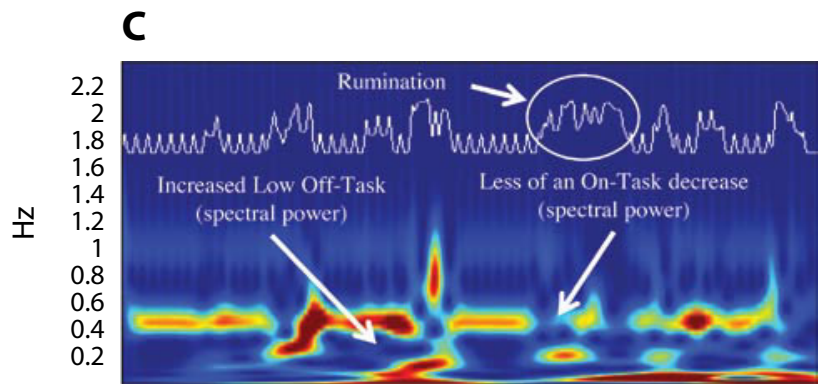

Time

\section{$\mathbf{E}$}



Time
B
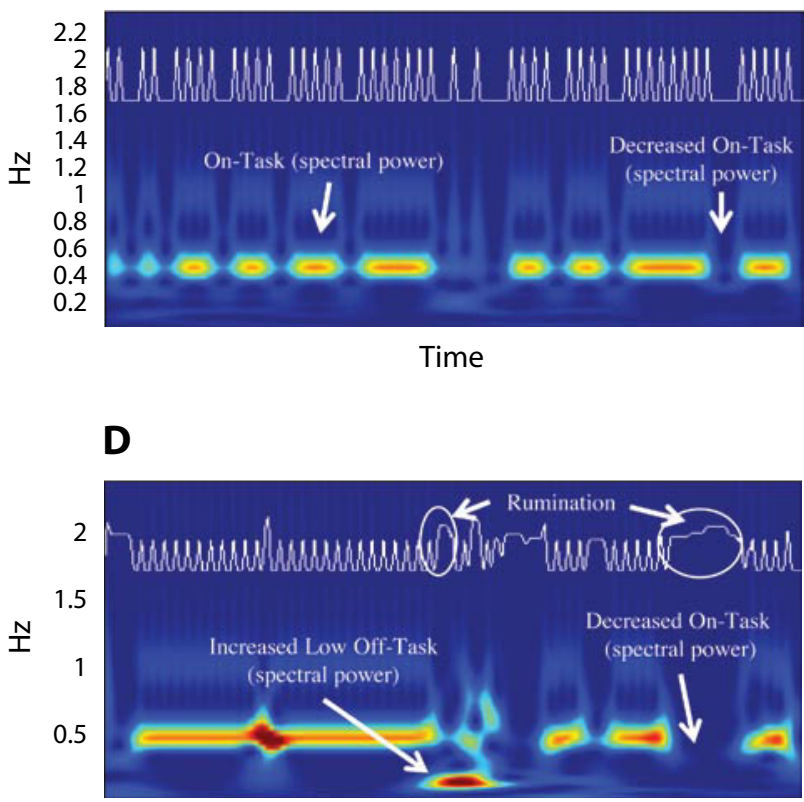

Time

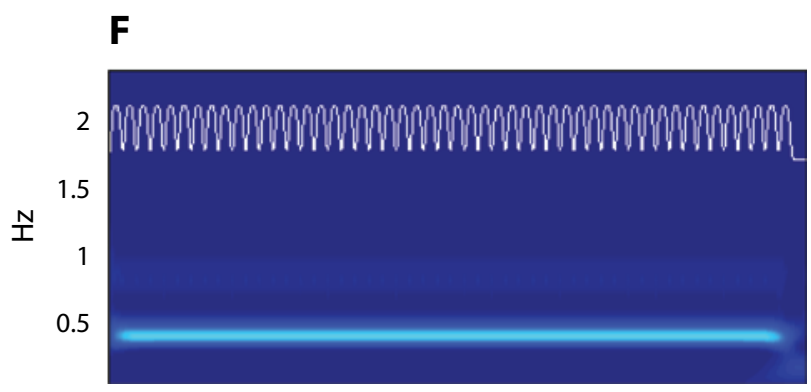

Time

Figure 1. (A) Simulated pupillary response with no lapses in attention. The solid white band indicates strong spectral power at $0.5 \mathrm{~Hz}$. In the color version of the figure, located online, the yellow and red colors indicate strong spectral power at $0.5 \mathrm{~Hz}$. (B) Simulated attentional lapses. Gaps in the solid white band at $0.5 \mathrm{~Hz}$ indicate decreased spectral power at $0.5 \mathrm{~Hz}$. Gaps in the yellow and red band at $0.5 \mathrm{~Hz}$ indicate decreased spectral power at $0.5 \mathrm{~Hz}$ (color version). (C) Simulated ruminative thoughts entailing trying to do the task. White bands and black spaces enclosed in white represent high spectral power. Yellow and red colors represent high spectral power (color version). When rumination occurs, there is increased spectral power $<0.37 \mathrm{~Hz}$ and increased spectral power at $0.5 \mathrm{~Hz}$. (D) Simulated rumination entailing not doing the task. White bands and black spaces enclosed in white represent high spectral power. Yellow and red colors represent high spectral power (color version). When rumination occurs, there is increased spectral power $<0.37 \mathrm{~Hz}$ and decreased spectral power at $0.5 \mathrm{~Hz}$. (E) Impulse response used to simulate slowed delayed response to stimuli. (F) Simulated power spectrum associated with slowed delayed responses.

were taking psychotropic medications, primarily SSRIs, but also mood stabilizers, antipsychotics, antianxiolytics, buproprion $\mathrm{HC}$, and tricyclic antidepressants (see Table 1), and medications for high blood pressure, insomnia, and birth control. Controls were recruited through flyers posted around the community advertising a study for never-depressed individuals, or they were contacted from an existing database of individuals who had previously signed consent to be recontacted for future studies in our lab. Controls had no current or historical Axis I disorder, which was determined by using the SCID-I interview. All of the participants scored within the normal range on the national adult reading test (NART-II VIQ $>80$; Nelson \& Willison, 1991) and had normal corrected vision (20/30 using a handheld eye chart). The depressed participants were significantly older and engaged in significantly higher levels of rumination than did controls (see Table 2). The distribution of gender between the groups did not differ $\left(\chi^{2}=0.921, p=.337\right)$, and the groups did not differ either in estimates of verbal intelligence or in psychomotor speed (as was indexed by their RTs during the PASAT).

\section{Apparatus}

A computer-based version of the PASAT was presented on a monitor that was approximately $65 \mathrm{~cm}$ away from the participant. Auditory stimuli were presented using four surround-sound speakers attached to the PC. Pupillary data were collected at $60 \mathrm{~Hz}$ (every $16.7 \mathrm{msec}$ ) in a moderately lit room (0.56-fc illuminance); the lighting was held constant across participants using a table-mounted ISCAN, Inc., RK726 video-based infrared pupilometer. The equipment digitized the video image and calculated the diameter of the pupil. 


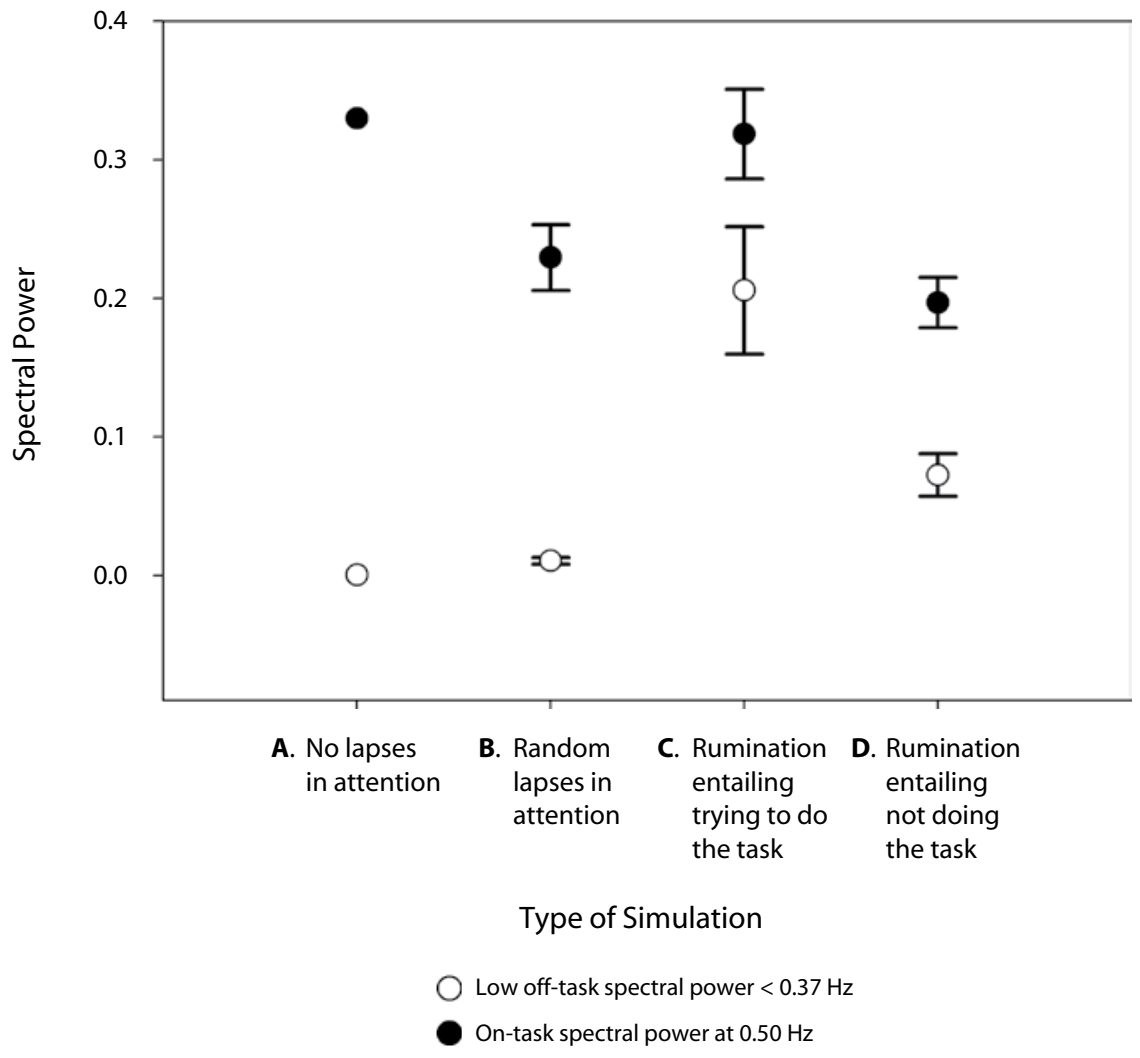

Figure 2. Mean differences in on-task and off-task spectral power across simulations.

\section{Procedure}

Participants were recruited to participate in a larger study assessing the efficacy of a cognitive control training intervention that was theorized to increase prefrontal function and decrease rumination (see Siegle, Ghinassi, \& Thase, 2007). The presented data are taken only from the initial two assessments prior to participants' engaging in the intervention. During an initial assessment, participants signed consent forms and received an SCID-I (First et al., 1996). In addition, participants completed a vision test and the NART-II (Nelson \& Willison, 1991). On a subsequent day, participants returned to the lab and completed the PASAT (Gronwall, 1977), which was followed by an assessment of mood and rumination. ${ }^{1}$ The PASAT was originally administered to collect a baseline of the subjects' prefrontal control in the context of the larger study. Pupil data were collected simultaneously during the completion of the PASAT.

\section{Tasks}

A computer-based version of the PASAT (Gronwall, 1977) was used in the present investigation. During the task, a series of numbers from 1 to 9 were administered via a digital recording emanating randomly from four different quadrants surrounding the participant. Participants were asked to (1) add the number presented to the number immediately preceding it, (2) enter the calculated sum via a mouse pointer by pressing the numbered buttons $1-18$ displayed in a circular pattern on-screen, and then (3) pay attention to the next incoming number of the auditory series to repeat the process. For example, given the series 1, 4, 3, a participant would add the pair " 1,4 ," click the response " 5 ," add the next number " 3 " to the previously presented number " 4 ," click the response " 7 ," and so on. Participants were told, "Don't worry if you make a mistake or leave some out. I want to see not only how long you can keep going without stopping but also how quickly you can pick up again if you do stop." Consistent with the standard PASAT, four 3-min blocks of increasing task difficulty were administered by reducing the interstimulus interval (ISI) in consecutive blocks from $2,400 \mathrm{msec}$ ( 74 addition trials in $180 \mathrm{sec}$, each lasting $2,400 \mathrm{msec}$ from the onset of one stimulus to the onset of the next) to $2,000 \mathrm{msec}$ ( $~ 90$ addition trials $/ 180 \mathrm{sec})$ to $1,600 \mathrm{msec}(\sim 113$ addition trials $/ 180 \mathrm{sec})$ to $1,200 \mathrm{msec}(\sim 150$ addition trials/180 sec). Participants were given at least a 30 -sec break between blocks. During the task, participants' trial-by-trial performance was displayed continuously on-screen in the form of the total number of responses correct, missed, and incorrect. This testing period was preceded by a short practice period at 2,400 msec lasting approximately $30 \mathrm{sec}$. Given that faster rates during the PASAT tend to be less sensitive to between-groups differences than are slower rates (Tombaugh, 2006, p. 55), we decided a priori to analyze the $2,400-$ msec block. $^{2}$

\section{Measures}

National Adult Reading Test (NART-II). The NART-II (Nelson \& Willison, 1991) was administered to be sure that participants had the cognitive and linguistic ability to complete the proposed task. The NART-II is a quick cognitive screening measure requiring minimal effort. It involves asking individuals to pronounce a list of 50 words. The test has been shown to have high correlations with WAIS-R verbal intelligence quotient (VIQ) scores $(r=.83)$ and is appropriate for use with depressed individuals because it is not affected by poor concentration or motivation.

Rumination/Reflection Questionnaire (RRQ). The RRQ (Trapnell \& Campbell, 1999) was used to assess the trait rumination. The 12-item rumination subscale assesses negative recurrent thoughts about the self that are prompted by threats, losses, or injustices to the self (e.g., "I tend to 'ruminate' or dwell over things that happen to me for a really long time afterward"). Items are rated on a 5-point scale ranging from 1 (strongly disagree) to 5 (strongly agree). Previous studies have demonstrated convergent validity 
Table 1

Demographic and Medication Status of Participants

\begin{tabular}{|c|c|c|c|c|}
\hline & \multicolumn{2}{|c|}{$\begin{array}{l}\text { Controls } \\
(n=19)\end{array}$} & \multicolumn{2}{|c|}{$\begin{array}{l}\text { Depressed } \\
(n=56)\end{array}$} \\
\hline & Frequency & $\%$ & Frequency & $\%$ \\
\hline \multicolumn{5}{|l|}{ Sex } \\
\hline Female & 15 & 79 & 37 & 67 \\
\hline \multicolumn{5}{|l|}{ Ethnicity } \\
\hline Caucasian & 15 & 79 & 44 & 80 \\
\hline African-American & 2 & 10.5 & 11 & 20 \\
\hline Asian & 2 & 10.5 & 0 & 0 \\
\hline \multicolumn{5}{|l|}{ Medications } \\
\hline SSRIs & 0 & 0 & 39 & 70 \\
\hline Mood stabilizers & 0 & 0 & 10 & 18 \\
\hline Antipsychotics & 0 & 0 & 5 & 9 \\
\hline Antianxiolytics & 0 & 0 & 14 & 25 \\
\hline Tricyclic antidepressants & 0 & 0 & 1 & 2 \\
\hline Buproprin HC & 0 & 0 & 8 & 14 \\
\hline
\end{tabular}

Note-SSRIs, selective serotonin reuptake inhibitors.

(Trapnell \& Campbell, 1999) and good internal reliability $(\alpha=.90)$ (Jones, Papadakis, Hogan, \& Strauman, 2009; Segerstrom, Stanton, Alden, \& Shortridge, 2003; Trapnell \& Campbell, 1999), and 3 -week test-retest stability $(r=.80)$ (Takano \& Tanno, 2009). ${ }^{3}$

\section{Data Selection, Cleaning, and Reduction}

Calculation of pupil dilation indices. The data were cleaned using methodology previously described (Siegle, Steinhauer, \& Thase, 2004). Briefly, following linear interpolation through blinks, data were smoothed using a 5-point unweighted average filter that was applied twice. Linear trends in pupil dilation calculated over experimental blocks were then removed from pupil dilation data to eliminate the effects of slow drift in pupil diameter. Pupil dilation in response to each trial was calculated by subtracting the pupil diameter in the $60 \mathrm{msec}$ preceding stimulus onset from the pupillary response throughout the trial. These data were used to calculate a measure of peak pupil dilation for correct and incorrect trials in order to examine the effect of drugs on the pupil.

Wavelet transformation. The cleaned pupil data were also subjected to a continuous wavelet transform algorithm to calculate the wavelet power spectrum using the "wavelet" function from Torrence and Compo's (1998) wavelet toolkit for MATLAB (The MathWorks, Inc., Natick, MA). A Morlet wavelet (wavenumber $\mathrm{w}_{0}=6$ ) was chosen as the mother wavelet, given its resemblance to the pupillary impulse response. Wavelet spectral power was normalized by dividing each band by its spectral frequency so that equivalent magnitude sine waves at any frequency were considered to have equivalent spectral power. This was done to control for the phenomenon that for the same magnitude of time-domain response, wavelet power in high frequencies is actually lower than wavelet power at lower frequencies. On the basis of our design, mean wavelet spectral power was calculated at $0.417 \mathrm{~Hz}$ for ISI 2,400 -msec trials; that is, we cal- culated the mean spectral power for each participant at the frequency at which the task was occurring (henceforth "on-task" power, our index of task-related resource allocation). Alternately, for each participant, we calculated the mean wavelet spectral power at and below $0.37 \mathrm{~Hz}$; that is, we calculated the mean spectral power in frequency bands occurring slower than the frequency at which the task was occurring (henceforth, "off-task" power, our index of off-task resource allocation). Given that the task occurred at $0.417 \mathrm{~Hz}$, we chose to calculate spectral power at and below $0.37 \mathrm{~Hz}$ to minimize the overlap between our measures of off-task resource allocation and on-task resource allocation. Power above the stimulus frequency was not examined, since it could reflect more transient processes and physiological artifacts.

Performance. Performance for each trial was captured by the PASAT software. Missing responses and wrong responses were coded as incorrect responses. Late responses, defined as providing a correct answer to the previous trial on the current trial, were not analyzed; that is, they were coded neither as errors nor as correct trials. There were no differences between depressed participants and controls in terms of the number of late responses. Complete analyses of late trials, along with their relationship to physiological reactivity, are available from the authors upon request. Errors were used to calculate the probability that a participant would make consecutive errors during the task. The probability of making consecutive errors - that is, $p\left(\right.$ error $_{t} \mid$ error $\left._{t-1}\right)$ - was calculated as the number of errors made immediately after an error during a block, divided by the total number of errors during the block. ${ }^{4}$

\section{RESULTS}

\section{Performance}

The task was sufficiently difficult $(M$ errors $=55.9$, $S D=17.0$, out of 75 trials) to yield interpretable analyses of consecutive errors. Depressed participants made more consecutive errors $\left[M p\left(\right.\right.$ error $_{t} \mid$ error $\left.\left._{t-1}\right)=.46, S D=.23\right]$ than did controls $(M=.30, S D=.18)(b=.16, S E=$ $.057, \beta=.31)[t(73)=-2.82, p=.006, d=0.79]$.

Associations of pupillary frequency measures with performance. We next examined whether pupillary measures that were hypothesized to index neural substrates of resource allocation were associated with performance. At the zero-order level, off-task power was significantly correlated with on-task processing $(r=.80, p<.0005)$ and with $p\left(\right.$ error $_{t} \mid$ error $\left._{t-1}\right)(r=.26, p=.023)$ (see Figure 3 ). However, on-task power was not significantly correlated with $p\left(\right.$ error $_{t} \mid$ error $\left._{t-1}\right)(r=.06, p=.586)$. Given the imprecise nature of our index of off-task processing, this index may also reflect some degree of on-task resource allocation-for example, being delayed in one's ability to produce the sum to a current trial. To clarify the relationship between on-task and off-task power with

Table 2

Group Differences in Age, Intelligence, and Motor Speed

\begin{tabular}{|c|c|c|c|c|c|c|}
\hline & \multicolumn{2}{|c|}{ Controls } & \multicolumn{2}{|c|}{ Depressed } & \multirow[b]{2}{*}{$t$ Value } & \multirow[b]{2}{*}{$d$} \\
\hline & $M$ & $S D$ & $M$ & $S D$ & & \\
\hline Age & 33.1 & 12.5 & 39.4 & 10.2 & 2.20 & $0.56^{*}$ \\
\hline VIQ (NART-II) & 111.3 & 9.6 & 111.1 & 7.8 & 0.06 & 0.02 \\
\hline Rumination (RRQ) & 28.7 & 7.7 & 47.2 & 9.4 & 7.74 & $2.16^{* * *}$ \\
\hline Reaction time ( $\mathrm{sec}$ ) & 1.64 & 0.20 & 1.66 & 0.28 & 0.26 & 0.07 \\
\hline
\end{tabular}

Note-VIQ, Estimated Verbal Intelligence Quotient; NART-II, National Adult Reading Test; RRQ, Rumination/Reflection Questionnaire. ${ }^{*} p<.05 . \quad{ }^{* * *} p<.0001$. 


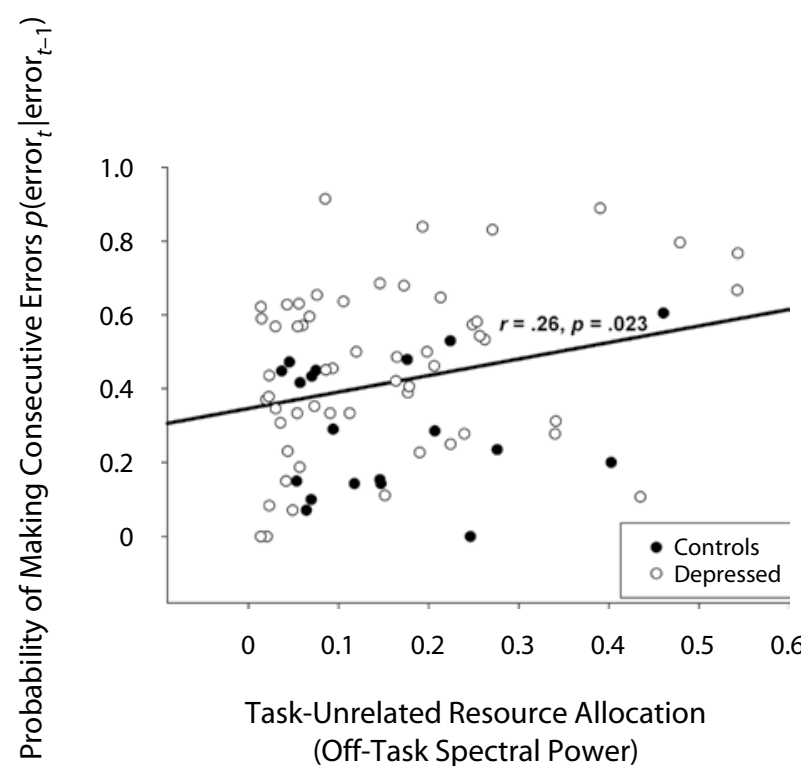

Figure 3. Association between off-task spectral power and cognitive performance.

performance, a hierarchical regression analysis was conducted $\left[F(2,72)=5.26, p=.007, R^{2}=.13\right]$. As was expected, off-task power was associated with a greater $p\left(\right.$ error $_{t} \mid$ error $\left._{t-1}\right)\left(b=1.00, S E=.314, \beta=.59, s r^{2}=\right.$ $.12, p=.002)$, and on-task power was associated with lower $p\left(\right.$ error $_{t} \mid$ error $\left._{t-1}\right)(b=-.96, S E=.433, \beta=-.41$, $\left.s r^{2}=.06, p=.031\right)$. Off-task power did not interact with on-task power to predict $p$ (error $\mid$ error $\left._{t-1}\right)\left[\Delta R^{2}=.00\right.$, $t(1)=0.17, p=.868]$.

This pattern of results indicates the presence of a suppressor situation (Conger, 1974; Tzelgov \& Henik, 1991); that is, the simultaneous inclusion of two predictors improves one or both zero-order correlations with the dependant variable (Paulhus, Robins, Trzesniewski, \& Tracy, 2004). This situation was bound to occur, given the highly collinear nature of our measures of on- and off-task power: Any differential effects could be revealed only when the shared variance was accounted for statistically. We used the Sobel test to evaluate the significance of these suppressor effects (MacKinnon, Krull, \& Lockwood, 2000). The effect of ontask power on PASAT performance significantly increased when off-task power was added to the equation $(z=2.16$, $p=.030$ ), and the effect of off-task power on PASAT performance significantly increased when on-task power was added to the equation $(z=3.07, p=.002)$. These results suggest that the allocation of cognitive resources to intrinsic processing, especially after accounting for the degree to which participants allocated resources to the cognitive task, significantly predicts poor performance.

\section{Test to Determine Whether Resource Allocation Explains Group Differences in Performance}

Next, we examined whether or not differences in performance between the controls and depressed participants were accounted for by the degree to which the participants allocated cognitive resources to off-task processing and/or on-task processing.

Group differences in on-task and off-task spectral power. Classical mediation analysis (Baron \& Kenny, 1986) can be used to examine the extent to which variance in performance that is associated with depression is accounted for by group differences in spectral power, although we were not formally testing a causal relationship. Our previous analysis satisfied the first step in determining mediation - that is, showing that depression status is associated with poorer cognitive performance. Next, we examined whether or not the groups differed on ontask and off-task spectral power (mediators). Given the high degree of overlap between these two constructs, we controlled for the alternate measure of resource allocation subsequent to examining group differences using multiple regression analyses. The overall model predicting offtask power from depression status controlling for on-task power was significant $\left[F(2,72)=73.92, p<.0001, R^{2}=\right.$ .67]. The depressed group (least-squares $M=.17$ ) had greater off-task power than did the controls (least-squares $M=.11)\left(b=.05, S E=.021, \beta=.18, s r^{2}=.03, p=\right.$ $.010)$ after controlling for on-task power $(b=1.16, S E=$ $\left..096, \beta=.84, s r^{2}=.67, p<.0001\right)$. Similarly, the overall model predicting on-task power from depression status controlling for off-task power was significant $[F(2,72)=$ $\left.79.92, p<.0001, R^{2}=.69\right]$. The depressed group (leastsquares $M=.10$ ) had less on-task power than did controls (least-squares $M=.15)(b=-.05, S E=.014, \beta=-.22$, $\left.s r^{2}=.05, p=.001\right)$ after controlling for off-task power $\left(b=.58, S E=.048, \beta=.80, s r^{2}=.64, p<.0001\right)$. These results suggest that depressed participants allocated fewer resources to the task and more cognitive resources to off-task processing, satisfying the second criteria for mediation (Baron \& Kenny, 1986). In our final two steps to test for mediation (Baron \& Kenny, 1986; MacKinnon, Lockwood, Hoffman, West, \& Sheets, 2002), we examined (1) whether off-task power (or on-task power) controlling for on-task power (or off-task power) and depression status predicted cognitive performance, and (2) whether the magnitude of the difference in performance between the depressed group and controls was significantly reduced after accounting for both on-task and off-task power.

Test of the magnitude of the reduced effect of depression status on cognitive performance. A multiple regression analysis was conducted predicting $p\left(\right.$ error $_{t} \mid$ error $\left._{t-1}\right)$ from depression status controlling for off-task and on-task power. The overall model was significant $\left[F(3,71)=5.44, p<.0001, R^{2}=.19\right]$. The depressed group (least-squares $M=.45$ ) remained significantly more likely than the controls to commit consecutive errors (least-squares $M=.31)(b=.13, S E=.059, \beta=.26$, $\left.s r^{2}=.06, p=.026\right)$ after controlling for off-task power $\left(b=.79, S E=.319, \beta=.46, s r^{2}=.07, p=.016\right)$ and ontask power $\left(b=-.58, S E=.453, \beta=-.24, s r^{2}=.02\right.$, $p=.207)$. The variance in performance accounted for by depression was slightly reduced when off-task power was accounted for using a Sobel test $(\Delta b=.03, z=1.80, p=$ $.072, p_{\text {one-tailed }}=.036$; see Figure 4 ). These results suggest that off-task power was a partial mediator of the effect of 


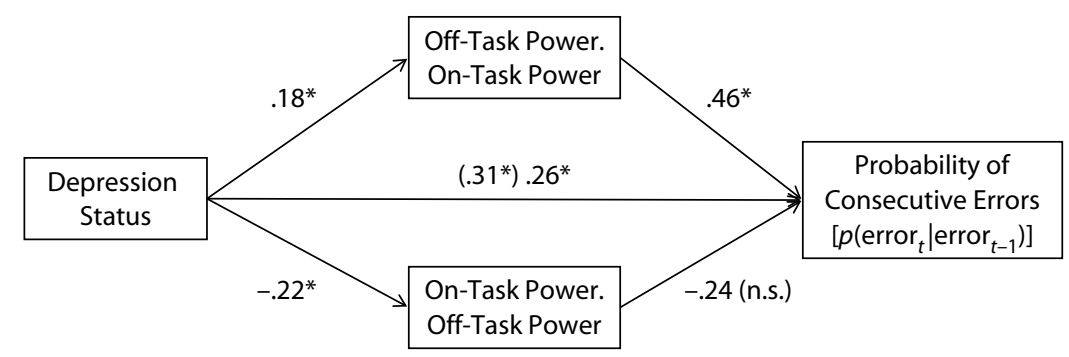

Figure 4. Model of the relationship among depression status, on-task power, offtask power, and the probability of making consecutive errors. The values presented are standardized regression coefficients. The value in parentheses represents the coefficient for the direct (i.e., unmediated) path. Depression status was coded as $0=$ controls, 1 = depressed. Off-task power.on-task power represents the effects of offtask power controlling for on-task power, and vice versa for on-task power.off-task power. ${ }^{*} p<.05$.

depression status on performance. In contrast, given that on-task power was not a significant predictor of performance after controlling for depression status and on-task power, it failed to pass this criterion for mediation.

\section{Continuous Associations of Spectral Power Indices With Rumination}

In an attempt to understand what our measure of offtask cognitive processing could be capturing, we examined the association between our power indices with the trait tendency to engage in rumination. Off-task and on-task power were not significantly correlated with rumination at the univariate level $(r=.13, p=.272$, and $r=-.06$, $p=.581$, respectively). Multiple regression analysis with on-task and off-task power as predictors and rumination as the dependent variable revealed significant relationships from the variables taken as a set $[F(2,72)=3.76$, $\left.p=.028, R^{2}=.09\right]$. Off-task power was positively associated with rumination $\left(\beta=.50, s r^{2}=.09, p=.009\right)$, and on-task power was negatively associated with rumination $\left(\beta=-.47, s r^{2}=.08, p=.015\right)$. Off-power and on-task power did not significantly interact to predict rumination $\left[\Delta R^{2}=.00, t(1)=-0.44, p=.664\right]$. Again, this pattern of results indicates that differential results could be observed only after accounting for the high level of multicolinearity that is not uniquely associated with either on- or off-task-related processes. The effect of on-task power on rumination increased significantly when off-task power was added to the equation $(z=2.60, p=.009)$, and the effect of off-task power on rumination increased significantly when on-task power was added to the equation $(z=$ $-2.43, p=.015)$. After controlling for depression diagnosis in the previous model, the overall model was significant $\left[F(3,71)=21.00, p<.0001, R^{2}=.47\right]$, although the effects for on-task power $\left(\beta=-.06, s r^{2}=.00\right)$ and off-task power $\left(\beta=.18, s r^{2}=.01\right)$ were nonsignificant $(p \mathrm{~s}>.1)$. This was because the depression diagnosis accounted for $38 \%$ of the variance in self-reported rumination $\left(\beta=.66, s r^{2}=.38, p<.001\right)$, leaving little residual variance to be accounted for by a physiological measure. Depression status did not interact with on-task or offtask power to predict rumination $\left[\Delta R^{2}=.00, F(2,69)=\right.$
$1.34, p=.268]$. Trait rumination was not associated with $p\left(\right.$ error $_{t} \mid$ error $\left._{t-1}\right)(r=.15, p=.186)$. These results show that individuals who are high in trait rumination demonstrate increased allocation of cognitive resources to offtask processing and decreased allocation of cognitive resources to on-task processing.

Influences of medication on pupillary responses. The following analyses examined whether taking medications with anticholinergic effects (e.g., olanzapine) affected the pupillary response. We examined the degree to which peak pupil dilation in response to correct and incorrect trials differed between depressed patients taking medications with anticholinergic effects $(n=18)$ and those who were not $(n=38)$. There were no statistical differences between the two groups on peak pupil dilation for correct trials $[t(54)=-1.15, p=.255, d=0.33]$ or for incorrect trials $[t(54)=-0.81, p=.420, d=0.22]$. These results indicate that medications had no detectable effect on pupillary responses.

Importantly, regression diagnostics indicated that the high degree of multicollinearity between on-task and offtask spectral power did not significantly inflate the variances of the regression coefficients in any analysis that contained both variables as predictors (all variance inflation factors were $<10$; Stevens, 2002, p. 92). In addition, no influential data points were observed, as determined by Cook's distance (Stevens, 2002, p. 132). Furthermore, we reconducted all major analyses controlling for age: The results did not significantly change any of the study's statistical effects or conclusions.

\section{DISCUSSION}

The primary objective of the present study was to clarify the mechanisms underlying cognitive deficits in depression. We specifically examined whether the likelihood of making consecutive errors on a cognitive task (PASAT) in depressed participants could be explained by either decreased allocation of cognitive resources to the task and/or increases in other potentially intrinsic processes that were not as clearly related to the task (e.g., negative automatic thoughts, emotional/ruminative processing, fatigue, etc.). 
Subsequently, we sought to clarify the nature of these lessstimulus-related processes by examining their association with trait rumination. In order to conduct this investigation, we calculated novel indices of cognitive resource allocation on the basis of pupil motility. Specifically, we created an index of task-related resource allocation on the basis of the assumption that pupillary motility occurring at the frequency of task stimuli reflects task-related cognitive function. In contrast, we assumed that pupil motility occurring at frequencies slower than the task would reflect the allocation of resources to slowly unfolding potentially intrinsic processes that were less clearly linked to each stimulus. We used computer simulations to generate predictions about how each of these processes would likely affect pupil motility (Figure 1).

As was predicted, depressed participants had a greater probability of making consecutive errors during the PASAT. This finding is consistent with previous investigations indicating that depressed individuals demonstrate error-related deficits on difficult cognitive tasks requiring cognitive control (Christopher \& MacDonald, 2005; Ottowitz et al., 2002), and that they demonstrate difficulty in responding to failure feedback during cognitive tasks (Elliott, Sahakian, Herrod, Robbins, \& Paykel, 1997; Elliott, Sahakian, McKay, \& Herrod, 1996; Holmes \& Pizzagalli, 2007).

Also consistent with predictions, to the extent that pupillary responsivity reflects neural substrates of performance, our data suggest that this psychophysiological measure could index resource allocation to task-related and less clearly task-related resource allocation. Allocating more resources that were not clearly tied to the stimuli was associated with decreased behavioral performance on the PASAT in both univariate and multivariate analyses. These findings are consistent with research demonstrating decreased performance during a continuous task requiring constant monitoring, as a result of increasing interference from an interspersed discrete task that vied for the same cognitive resources (Salvucci \& Taatgen, 2008).

In addition, our index of potentially intrinsic non-taskrelated physiological activity during the PASAT partially mediated the association between depression status and performance when using a one-tailed hypothesis test. However, allocating resources to task-related processing was not a significant mediator. Depressed participants in our sample allocated more cognitive resources to intrinsic processing than did the controls, who allocated more cognitive resources to the task. These findings suggest that one of the contributing factors to cognitive control deficits in depression is the allocation of cognitive resources to intrinsic processes. On the basis of our simulations (Figures $2 \mathrm{~B}$ and $2 \mathrm{~F}$ ), consecutive errors were likely not associated with either an absence of task-related resource allocation or with systematically sustained/delayed responses. Our results fit with emerging neuroimaging research indicating that depressed patients demonstrate increased activation in brain regions that are associated with affective and emotional processing, and decreased activation in the dorsolateral prefrontal cortex during dif- ficult cognitive tasks (Holmes \& Pizzagalli, 2008). These data are also consistent with research demonstrating that allocating processing resources to rumination during cognitive tasks that require controlled processing is associated with impaired performance (Lyubomirsky, Kasri, \& Zehm, 2003; Lyubomirsky \& Nolen-Hoeksema, 1995; Lyubomirsky, Tucker, Caldwell, \& Berg, 1999; Watkins \& Brown, 2002). Since depressed participants still performed worse than controls after accounting for both indices of resource allocation, other mediating factors - in addition to resource allocation - clearly contribute to poor cognitive performance, and these were not captured in the present investigation. For example, research suggests that cognitive deficits in depression may reflect deficits in motivation that can be remediated by encouraging patients to set goals related to enhancing their performance (Scheurich et al., 2008). Similarly, research has shown that individual differences in exposure to stress as indexed by cortisol secretions, specifically within depressed individuals, are associated with greater cognitive control deficits (Hinkelmann et al., 2009). In light of our findings, our posited resource allocation model of cognitive deficits in depression would benefit from being expanded to incorporate individual differences in depression, such as exposure to stress and motivational impairment (Carragher, Adamson, Bunting, \& McCann, 2009; Chen, Eaton, Gallo, \& Nestadt, 2000; Manji, Drevets, \& Charney, 2001; Nestler \& Carlezon, 2006).

Because we did not measure self-reported rumination during the task, we were unable to further validate that our measure of intrinsic processing was directly related to rumination during the task. However, we found modest evidence linking greater resource allocation to intrinsic processing with higher levels of the trait tendency to engage in rumination; the converse was true for task-related resource allocation. This finding could suggest that intrinsic processing in our study may be capturing ruminative thought processes. However, this interpretation is further tempered by the fact that trait rumination was unrelated to performance. In spite of this limitation, our results are consistent with existing cognitive theories of depression and self-regulatory theories of depression that hypothesize that perceived failure can initiate or exacerbate negatively biased cognitions, and possibly negative affect, that derail subsequent performance (Beck, 1963, 1970, 1987; Pyszczynski \& Greenberg, 1987; Teasdale, 1988).

Other limitations of the study warrant discussion. First, the majority of participants were on antidepressant medication $(70 \%)$ that is known to cause reductions in rumination (Bagby et al., 1999; Schmaling, Dimidjian, Katon, \& Sullivan, 2002). This could have resulted in an attenuation of the natural tendency to engage in rumination, resulting in being less off-task and thereby reducing the magnitude of our findings. Similarly, since all participants were recruited from an intensive outpatient unit that included psychotherapy, pharmacotherapy, and the potential for participation in our cognitive control training intervention directed specifically at rumination, they may have been sensitized to the role that their cognitions- 
and, specifically, rumination - play in their performance, thus limiting our study's generalizability. Second, the observed partial mediation effects were marginally significant when examined using a two-tailed hypothesis test. A one-tailed test was warranted, given that hypotheses and formulation (change in variance accounted for) could detect only reductions that were associated with the mediator variable. Notably, detecting significant effects with twotailed tests using this technique requires notoriously high power (MacKinnon et al., 2002); we would have needed approximately 422 subjects to be statistically powered at .80 to detect our effects using the Sobel test (Fritz \& MacKinnon, 2007). Third, the power of pupil diameter variation at frequencies below $0.8 \mathrm{~Hz}$ has been reliably used to measure sleepiness (Wilhelm et al., 2001; Wilhelm, Wilhelm, Ludtke, Streicher, \& Adler, 1998). Our pupillary indices of task-related and intrinsic processing are below the $0.8-\mathrm{Hz}$ frequency range, suggesting that our results could potentially be confounded with sleepiness, which was not directly assessed. That said, there is not much data to suggest that sleepiness effects on the pupil are detectable during an engaging cognitive task. Last, we did not directly assess motivation or task disengagement during the PASAT, which may have refined our ability to detect group differences in behavioral performance.

These limitations notwithstanding, the present investigation contributes to existing literature through the development of novel physiological indices of task-related and potentially intrinsic information processing and its use in understanding information processing in depression. This was accomplished without overtly disrupting the concurrent investigation of controlled cognitive processing. Our results support the idea that wavelet analyses of pupillary motility reliably index cognitive states. Furthermore, cognitive deficits in depression appear to involve primarily increased intrinsic processing that may be associated with rumination.

There are multiple exciting future directions suggested by the present study. In conjunction with neuroimaging, this methodology could aid in the detection of brain mechanisms that are associated with cognitive deficits in subsets of depressed individuals. This technique may be a particularly useful tool for assessing posited mechanisms of change in interventions that target ruminative processes, such as cognitive therapy (Beck, 1979) or Wells's (2000) attention training. Our results indicate that accounting for the effects of both task-related and intrinsic processing, such as affective arousal, rumination, and potentially motivation, even in response to nominally nonemotional stimuli, are important when gaining a more complete understanding of cognitive functioning deficits in depression.

\section{AUTHOR NOTE}

We gratefully acknowledge contributions of the volunteers who participated in this study, as well as Lisa Farace and Dimple Sodhi for contributions to data collection, and the staff of WPIC's Intensive Outpatient Program for their help in recruiting for this study. The present research was supported by MH64159, MH082998, NARSAD, and MH30915. Address correspondence to N. P. Jones, Western Psychiatric Institute and Clinic, University of Pittsburgh, 3811 O'Hara St., Pittsburgh, PA 15213 (e-mail: jonesnp@upmc.edu).

\section{REFERENCES}

Audenaert, K., Goethals, I., Van Laere, K., Lahorte, P., Brans, B., VERSIJPT, J., ET AL. (2002). SPECT neuropsychological activation procedure with the Verbal Fluency Test in attempted suicide patients. Nuclear Medicine Communications, 23, 907-916.

Austin, M., Mitchell, P., \& Goodwin, G. M. (2001). Cognitive deficits in depression: Possible implications for functional neuropathology. British Journal of Psychiatry, 178, 200-206.

Bagby, R. M., Rector, N. A., Segal, Z. V., Joffe, R. T., Levitt, A. J., Kennedy, S. H., \& Levitan, R. D. (1999). Rumination and distraction in major depression: Assessing response to pharmacological treatment. Journal of Affective Disorders, 55, 225-229.

BARON, R. M., \& KeNNY, D. A. (1986). The moderator-mediator variable distinction in social psychological research: Conceptual, strategic, and statistical considerations. Journal of Personality \& Social Psychology, 51, 1173-1182.

BeAtTy, J. (1982a). Phasic not tonic pupillary responses vary with auditory vigilance performance. Psychophysiology, 19, 167-172.

BeatTy, J. (1982b). Task-evoked pupillary responses, processing load, and the structure of processing resources. Psychological Bulletin, 91, 276-292.

BECK, A. T. (1963). Thinking and depression: Idiosyncratic content and cognitive distortions. Archives of General Psychiatry, 9, 324-333.

BECK, A. T. (1970). Cognitive therapy: Nature and relation to behavior therapy. Behavior Therapy, 1, 184-200.

Beck, A. T. (1979). Cognitive therapy of depression. New York: Guilford.

BECK, A. T. (1987). Cognitive models of depression. Journal of Cognitive Psychotherapy, 1, 5-37.

Carragher, N., Adamson, G., Bunting, B., \& McCann, S. (2009). Subtypes of depression in a nationally representative sample. Journal of Affective Disorders, 113, 88-99.

Chen, L.-S., Eaton, W. W., Gallo, J. J., \& Nestadt, G. (2000). Understanding the heterogeneity of depression through the triad of symptoms, course and risk factors: A longitudinal, population-based study. Journal of Affective Disorders, 59, 1-11.

Christopher, G., \& MacDonald, J. (2005). The impact of clinical depression on working memory. Cognitive Neuropsychiatry, 10, 379399.

Conger, A. J. (1974). A revised definition for suppressor variables: A guide to their identification and interpretation. Educational \& Psychological Measurement, 34, 35-46.

Elliott, R., Baker, S. C., Rogers, R. D., O'Leary, D. A., Paykel, E. S., ET AL. (1997). Prefrontal dysfunction in depressed patients performing a complex planning task: A study using positron emission tomography. Psychological Medicine, 27, 931-942.

Elliott, R., Sahakian, B. J., Herrod, J. J., Robbins, T. W., \& PAYKEL, E. S. (1997). Abnormal response to negative feedback in unipolar depression: Evidence for a diagnosis specific impairment. Journal of Neurology, Neurosurgery \& Psychiatry, 63, 74-82.

Elliott, R., Sahakian, B. J., McKay, A. P., \& Herrod, J. J. (1996). Neuropsychological impairments in unipolar depression: The influence of perceived failure on subsequent performance. Psychological Medicine, 26, 975-989.

Feldner, M. T., Leen-Feldner, E. W., Zvolensky, M. J., \& Lejuez, C. W. (2006). Examining the association between rumination, negative affectivity, and negative affect induced by a paced auditory serial addition task. Journal of Behavior Therapy \& Experimental Psychiatry, 37, 171-187.

First, M. B., Spitzer, R. L., Gibbon, M., \& Williams, J. B. (1996). Structured clinical interview for DSM-IV axis I disorders patient edition (Vol. 20). New York: New York State Psychiatric Institute, Biometrics Research Department.

Fish, S. C., \& GRANHOLM, E. (2008). Easier tasks can have higher processing loads: Task difficulty and cognitive resource limitations in schizophrenia. Journal of Abnormal Psychology, 117, 355-363.

Fitzgerald, P. B., SRithiran, A., Benitez, J., Daskalakis, Z. Z., Oxley, T. J., KulKarni, J., et al. (2008). An fMRI study of prefrontal brain activation during multiple tasks in patients with major depressive disorder. Human Brain Mapping, 29, 490-501.

Fritz, M. S., \& MacKinnon, D. P. (2007). Required sample size to detect the mediated effect. Psychological Science, 18, 233-239. 
Gow, A. J., \& DeAry, I. J. (2004). Is the PASAT past it? Testing attention and concentration without numbers. Journal of Clinical \& Experimental Neuropsychology, 26, 723-736.

Granholm, E., Asarnow, R. F., Sarkin, A. J., \& Dykes, K. L. (1996). Pupillary responses index cognitive resource limitations. Psychophysiology, 33, 457-461.

Gronwall, D. (1977). Paced auditory serial-addition task: A measure of recovery from concussion. Perceptual \& Motor Skills, 44, 367-373.

Harvey, P.-O., Fossati, P., Pochon, J.-B., Levy, R., Lebastard, G., LEHÉRICY, S., ET AL. (2005). Cognitive control and brain resources in major depression: An fMRI study using the n-back task. NeuroImage, 26, 860-869.

Hinkelmann, K., Moritz, S., Botzenhardt, J., Riedesel, K., Wiedemann, K., Kellner, M., \& Otte, C. (2009). Cognitive impairment in major depression: Association with salivary cortisol. Biological Psychiatry, 66, 879-885.

Holmes, A. J., \& Pizzagalli, D. A. (2007). Task feedback effects on conflict monitoring and executive control: Relationship to subclinical measures of depression. Emotion, 7, 68-76.

Holmes, A. J., \& Pizzagalli, D. A. (2008). Spatiotemporal dynamics of error processing dysfunctions in major depressive disorder. $\mathrm{Ar}$ chives of General Psychiatry, 65, 179-188.

Johnstone, T., van Reekum, C. M., Urry, H. L., Kalin, N. H., \& Davidson, R. J. (2007). Failure to regulate: Counterproductive recruitment of top-down prefrontal-subcortical circuitry in major depression. Journal of Neuroscience, 27, 8877-8884.

Jones, N. P., Papadakis, A. A., Hogan, C. M., \& Strauman, T. J. (2009). Over and over again: Rumination, reflection, promotion goal failure, and their interactive effects on depressive symptoms. Behavior Research \& Therapy, 47, 254-259.

JoORMANN, J., \& GoTLIB, I. H. (2008). Updating the contents of working memory in depression: Interference from irrelevant negative material. Journal of Abnormal Psychology, 117, 182-192.

Koikegami, H., \& Yoshida, K. (1953). Pupillary dilation induced by stimulation of amygdaloid nuclei. Folia Psychiatrica et Neurologica Japonica, 7, 109-126.

Lazeron, R. H. C., Rombouts, S. A. R. B., De Sonneville, L., BarkHof, F., \& Scheltens, P. (2003). A paced visual serial addition test for fMRI. Journal of the Neurological Sciences, 213, 29-34.

LyUbOMiRSKy, S., KASRI, F., \& ZEHM, K. (2003). Dysphoric rumination impairs concentration on academic tasks. Cognitive Therapy \& Research, 27, 309-330.

Lyubomirsky, S., \& Nolen-Hoeksema, S. (1995). Effects of selffocused rumination on negative thinking and interpersonal problem solving. Journal of Personality \& Social Psychology, 69, 176-190.

Lyubomirsky, S., Tucker, K. L., CAldwell, N. D., \& Berg, K. (1999). Why ruminators are poor problem solvers: Clues from the phenomenology of dysphoric rumination. Journal of Personality \& Social Psychology, 77, 1041-1060.

MacDonald, A. W., Cohen, J. D., Stenger, V. A., \& Carter, C. S. (2000). Dissociating the role of the dorsolateral prefrontal and anterior cingulate cortex in cognitive control. Science, 288, 1835-1838.

MacKinnon, D. P., Krull, J. L., \& Lockwood, C. M. (2000). Equivalence of the mediation, confounding and suppression effect. Prevention Science, 1, 173-181.

MacKinnon, D. P., Lockwood, C. M., Hoffman, J. M., West, S. G., \& Sheets, V. (2002). A comparison of methods to test mediation and other intervening variable effects. Psychological Methods, 7, 83-104.

Manji, H. K., Drevets, W. C., \& Charney, D. S. (2001). The cellular neurobiology of depression. Nature Medicine, 7, 541-547.

Marshall, S. P. (2007). Identifying cognitive state from eye metrics. Aviation, Space, \& Environmental Medicine, 78(Suppl. 5), B165-B175.

Matsuo, K., Glahn, D. C., Peluso, M. A. M., Hatch, J. P., Monkul, E. S., NAJT, P., ET AL. (2007). Prefrontal hyperactivation during working memory task in untreated individuals with major depressive disorder. Molecular Psychiatry, 12, 158-166.

Nelson, H. E., \& Willison, J. R. (1991). National Adult Reading Test (NART): Test manual (2nd ed.). Berkshire, U.K.: Nfer-Nelson.

Nestler, E. J., \& Carlezon, W. A. (2006). The mesolimbic dopamine reward circuit in depression. Biological Psychiatry, 59, 1151-1159.
Nolen-Hoeksema, S. (1991). Responses to depression and their effects on the duration of depressive episodes. Journal of Abnormal Psychology, 100, 569-582.

OKada, G., OKamoto, Y., Morinobu, S., \& Yamawaki, S. Y. N. (2003). Attenuated left prefrontal activation during a verbal fluency task in patients with depression. Neuropsychobiology, 47, 21-26.

Ottowitz, W. E., Tondo, L., Dougherty, D. D., \& Savage, C. R. (2002). The neural network basis for abnormalities of attention and executive function in major depressive disorder: Implications for application of the medical disease model to psychiatric disorders. Harvard Review of Psychiatry, 10, 86-99.

Paulhus, D. L., Robins, R. W., Trzesniewski, K. H., \& Tracy, J. L. (2004). Two replicable suppressor situations in personality research. Multivariate Behavioral Research, 39, 303-328.

Pizzagalli, D. A., Peccoralo, L. A., Davidson, R. J., \& Cohen, J. D. (2006). Resting anterior cingulate activity and abnormal responses to errors in subjects with elevated depressive symptoms: A 128-channel EEG study. Human Brain Mapping, 27, 185-201.

PyszCZYNSKI, T., \& GREENBERG, J. (1987). Self-regulatory perseveration and the depressive self-focusing style: A self-awareness theory of reactive depression. Psychological Bulletin, 102, 122-138.

RATCLIFF, R. (1976). A mathematical model for paced serial addition. Journal of Mathematical Psychology, 13, 16-78.

Ridderinkhof, K. R., Ullsperger, M., Crone, E. A., \& NieuwenHUIS, S. (2004). The role of the medial frontal cortex in cognitive control. Science, 306, 443-447.

Riso, L. P., du Toit, P. L., Blandino, J. A., Penna, S., Dacey, S., Duin, J. S., ET AL. (2003). Cognitive aspects of chronic depression. Journal of Abnormal Psychology, 112, 72-80.

Rogers, M. A., Kasai, K., Koji, M., Fukuda, R., Iwanami, A., NaKAGOME, K., ET AL. (2004). Executive and prefrontal dysfunction in unipolar depression: A review of neuropsychological and imaging evidence. Neuroscience Research, 50, 1-11.

Salvucci, D. D., \& TaAtgen, N. A. (2008). Threaded cognition: An integrated theory of concurrent multitasking. Psychological Review, 115, 101-130.

Scheurich, A., Fellgiebel, A., Schermuly, I., Bauer, S., WolfGES, R., \& MULLER, M. J. (2008). Experimental evidence for a motivational origin of cognitive impairment in major depression. Psychological Medicine, 38, 237-246.

Schmaling, K. B., Dimidian, S., Katon, W., \& Sullivan, M. (2002). Response styles among patients with minor depression and dysthymia in primary care. Journal of Abnormal Psychology, 111, 350-356.

Segerstrom, S. C., Stanton, A. L., Alden, L. E., \& Shortridge, B. E. (2003). A multidimensional structure for repetitive thought: What's on your mind, and how, and how much? Journal of Personality \& Social Psychology, 85, 909-921.

Shah, P., O'Carroll, R., Rogers, A., Moffoot, A., \& Ebmeier, K. (1999). Abnormal response to negative feedback in depression. Psychological Medicine, 29, 63-72.

Siegle, G. J., Ghinassi, F., \& Thase, M. E. (2007). Neurobehavioral therapies in the 21st century: Summary of an emerging field and an extended example of cognitive control training for depression. Cognitive Therapy \& Research, 31, 235-262.

Siegle, G. J., Granholm, E., Ingram, R. E., \& Matt, G. E. (2001). Pupillary and reaction time measures of sustained processing of negative information in depression. Biological Psychiatry, 49, 624-636.

Siegle, G. J., Moore, P. M., \& Thase, M. E. (2004). Rumination: One construct, many features in healthy individuals, depressed individuals, and individuals with lupus. Cognitive Therapy \& Research, 28, 645-668

Siegle, G. J., Steinhauer, S. R., Stenger, V. A., Konecky, R., \& CARTER, C. S. (2003). Use of concurrent pupil dilation assessment to inform interpretation and analysis of fMRI data. NeuroImage, 20, 114-124.

Siegle, G. J., Steinhauer, S. R., \& Thase, M. E. (2004). Pupillary assessment and computational modeling of the Stroop task in depression. International Journal of Psychophysiology, 52, 63-76.

Siegle, G. J., Thompson, W. S., Carter, C. S., Steinhauer, S. R., \& Thase, M. E. (2007). Increased amygdala and decreased dorsolateral prefrontal BOLD responses in unipolar depression: Related and independent features. Biological Psychiatry, 61, 198-209. 
Steinhauer, S. R., \& HaKerem, G. (1992). The pupillary response in cognitive psychophysiology and schizophrenia. Annals of the New York Academy of Sciences, 658, 182-204.

Stevens, J. (2002). Applied multivariate statistics for the social sciences (4th ed.). Mahwah, NJ: Erlbaum.

TAKANO, K., \& TANnO, Y. (2009). Self-rumination, self-reflection, and depression: Self-rumination counteracts the adaptive effect of selfreflection. Behaviour Research \& Therapy, 47, 260-264.

Teasdale, J. D. (1988). Cognitive vulnerability to persistent depression. Cognition \& Emotion, 2, 247-274.

Tombaugh, T. N. (2006). A comprehensive review of the Paced Auditory Serial Addition Test (PASAT). Archives of Clinical Neuropsychology, 21, 53-76.

Torrence, C., \& Compo, G. P. (1998). A practical guide to wavelet analysis. Bulletin of the American Meteorological Society, 79, 61-78.

Trapnell, P. D., \& Campbell, J. D. (1999). Private self-consciousness and the five-factor model of personality: Distinguishing rumination from reflection. Journal of Personality \& Social Psychology, 76, 284 304.

Tzelgov, J., \& HeniK, A. (1991). Suppression situations in psychological research: Definitions, implications, and applications. Psychological Bulletin, 109, 524-536.

Walter, H., Wolf, R. C., Spitzer, M., \& Vasic, N. (2007). Increased left prefrontal activation in patients with unipolar depression: An event-related, parametric, performance-controlled fMRI study. Journal of Affective Disorders, 101, 175-185.

Watkins, E., \& Brown, R. G. (2002). Rumination and executive function in depression: An experimental study. Journal of Neurology, Neurosurgery \& Psychiatry, 72, 400-402.

Wells, A. (2000). Emotional disorders and metacognition innovative cognitive therapy. New York: Wiley.

WICKENS, C. D. (2002). Multiple resources and performance prediction. Theoretical Issues in Ergonomics Science, 3, 159-177.

Wilhelm, B., Giedke, H., Ludtke, H., Bittner, E., Hofmann, A., \& Wilhelm, H. (2001). Daytime variations in central nervous system activation measured by a pupillographic sleepiness test. Journal of Sleep Research, 10, 1-7.

Wilhelm, B., Wilhelm, H., Ludtke, H., Streicher, P., \& Adler, M.
(1998). Pupillographic assessment of sleepiness in sleep-deprived healthy subjects. Sleep, 21, 258-265.

\section{NOTES}

1. During both the initial assessment and the subsequent lab visit, participants completed several other information processing and attention control tasks that were not examined in the present investigation.

2. The decision to analyze only the 2,400-msec data was made a priori. We did not feel that the other presentation rates were suitable to test our current hypothesis, given their level of difficulty and previous research suggesting that these presentation rates are not sensitive to finding group differences between patients with traumatic brain injuries and controls (Tombaugh, 2006). However, given that this was the first time we had used a software-based interface for responses, we were unsure whether we would have sufficient error rates at the 2,400-msec block to analyze; thus, we thought that it was best to give all three blocks. In light of the poor performance at even the 2,400-msec block, it was deemed acceptable for analysis.

3. Of note, a large battery of rumination measures were administered (Siegle, Moore, \& Thase, 2004). The RRQ was selected from these a priori as a measure likely to (1) dissociate symptomatology from rumination, and (2) specifically assess aspects of rumination that are associated with goal failure. For the present study, no other measures of rumination were analyzed, and there was no capitalization on chance results that were associated with one measure or another. Seven subjects were missing data on the RRQ; these missing responses were singly imputed from subjects' responses to other rumination measures. We also conducted post hoc analyses using other rumination measures to verify that the results were not dependent on the type of rumination measure used.

4. We also considered using a runs test (Shah, O'Carroll, Rogers, Moffoot, \& Ebmeier, 1999); however, this test does not distinguish between making correct and incorrect runs, and, in the present sample, the mean number of correct or incorrect runs was $<20$, indicating that this test was not appropriate.

(Manuscript received March 24, 2009; revision accepted for publication October 25, 2009.) 